\title{
SPACE MICRO-ROBOTS
}

Valentyn Pidvysotskyi, https://orcid.org/0000-0002-0028-757X

\begin{abstract}
The article proposes a method of dealing with space debris. This method is based on the use of a large number of controlled space micro-robots, which are moving in a retrograde near-earth orbit (towards the particles of space debris). Strategy of struggle space debris consist in targeting micro-robots at large and medium-sized objects to change their orbit (in case of a threat of collision). The task of such an impact is prevention the chain reaction of propagation of space debris because of the collision of these objects. Destruction of small particles of space debris will occur due at the expense of random collisions with micro-robots. The control system makes it possible to divert the micro-robots away from the operating spacecraft's. In addition, each micro-robot is equipped with a self-destruct system, which helps prevent collisions with the operating spacecraft in the event of a control system failure. It is shown that the new method allows cleaning the space corridor from small particles of space debris for the safe movement of spacecraft's in a short time and at low cost. In parallel with the elimination of space debris, the some additional possibilities of orbital grouping of micro-robots is being consider. For example, the orbital grouping of micro-robots can be used for detection and to refine the orbital characteristics of particles of space debris (i.e. perform the functions of an astronomical instrument and navigation system). Micro-robots can perform functions of orbit correction, deceleration, or acceleration of operating spacecraft. Considered the possibility of creating a fundamentally new space engine (using micro-robots as a propellant). It is shown that the new engine has high efficiency and can be used to launch spacecraft to Mars. This engine allows recycling a large number of old micro-robots (which must be removed from Earth orbit).
\end{abstract}

Keywords: Micro-robot; Kinetic; Explosion; Engine; Space; Debris; Spacecraft; Smart; Dust

\section{Nomenclature}

$R$ - circular orbit of radius

$d R$ - infinitely small change in the radius of the circular orbit

$R_{i}$ - initial radius of the circular orbit

$R_{f}$ - the final radius of the circular orbit

$v$ - oncoming speed of a particle of space debris and micro-robot

$\bar{v}$ - average oncoming speed of a particles of space debris and micro-robots

$d v$ - infinitely small change in counter speed

$v_{d}$ - space debris orbital speed

$v_{1}$ - first cosmic velocity

$v_{2}$ - second cosmic velocity

$M_{r}$ - mass of the micro-robot

$M_{d}$ - mass of the particle of space debris 
$t$ - period of time

$d t$ - infinitely small period of time

$\bar{n}$ - average amount of measurements of the coordinates of particles of space debris

$\omega$ - frequency of measurements of the coordinates of particles of space debris

$L$ - micro-radar range

$d E$ - infinitely small change in the total energy of the particle debris

$d E_{k}$ - infinitely small change in the kinetic energy of the particle debris

$d E_{p}$ - infinitesimal change in the potential energy of a particle of space debris

$\Delta S$ - debris particle cross-sectional area

$x$ - ratio of the cross-sectional area $\Delta S$ of debris particle to its own mass $M_{d}$

$N$ - concentration of micro-robots

$V$ - orbital corridor volume

$\sum M_{r}$ - total mass of micro-robots colliding with a particle of space debris

$M$ - total mass of micro-robots inside the orbital corridor

$d E_{k}$ - infinitely small change in the kinetic energy of the particle debris

$d E_{p}$ - infinitesimal change in the potential energy of a particle of space debris

$m$ - mass of the spacecraft

$d m$ - infinitesimal change in the mass of the spacecraft

$m_{i}$ - initial mass the spacecraft

$m_{f}$ - final mass of the spacecraft

$v_{s}$ - speed of the spacecraft

$v_{i}$ - initial oncoming speed spacecraft and micro-robots

$v_{f}$ - final oncoming speed spacecraft and micro-robots

$d M$ - mass of the gas portion (supplied to the working chamber)

$u$ - exhaust speed of the gas mixture (from the working chamber)

$\eta$ - engine coefficient of efficiency

$g_{0}$ - standard acceleration due to gravity on the surface of the Earth

$I_{s p}$ - specific impulse (s)

$n$ - coefficient of proportionality

$n_{\text {opt }}$ - the optimal value of the coefficient of proportionality $n$

$T_{o}$ - orbital period (for an elliptical orbit)

$a$ - semi-major axis (for an elliptical orbit)

$\mu$ - gravitational parameter 
$R_{p}$ - orbit pericenter distance to the center of the Earth

\section{Introduction}

The increase of the amount of space debris can becomes a serious problem due to increased collision frequency and of occurrence uncontrolled fission of particles of space debris [1]. This leads to the intensification of work in the field of struggle with space debris. Currently used and developed several main areas of the fight against space debris.

\subsection{Transfer of large objects to graveyard orbit}

After the operation is completed, the spacecraft is transferred to a graveyard orbit. This allows long-term storage of non-performing spacecraft, which may become the object of study of «space archaeologists». Over time, these burials can acquire greater value (material or cultural). It can be predicted that in the future programs will be developed to preserve and protect ancient space artifacts (in the form of remaining spacecraft and their parts).

The disadvantage of this method is the high costs of propellant for the transfer of the spacecraft to the graveyard orbit. In addition, in the future burial orbits may be needed for some other purpose. Prolonged accumulation of inoperative spacecraft's can lead to a local Kessler effect [1]. Therefore, eventually, the graveyard orbits can turn into another source of space debris.

\subsection{Appearance prevention of new space debris}

In many cases, after the operation is completed, the spacecraft can be braked by the propulsion system. In addition, for braking, can be use an inflatable sphere [2], sail [3; 4], foam sphere [5], electrodynamic tether [6; 7], electrostatic field [8] or others. As a result of braking, the orbit height decreases and the spacecraft will burn in the atmosphere of the Earth. The advantage is the complete elimination of the potential source of pollution of outer space.

\subsection{Destruction of existing space debris}

Currently, this area is developing most actively. There is a program for collecting garbage with the help of special networks [9; 10], a thin membrane [11], and a harpoon [12]. There are projects of impact on debris with the help of a laser [13;14;15], ion beam [16], and plasma flow [17]. There are projects for the destruction of garbage with tungsten micro-particles [18]. There are projects using space debris as a reactive mass [19] or energy source [20]. There is a project to capture space debris using glue [21], manipulators or other capture devices [22], etc. All these projects are workable and are of great interest. Practical application will allow comparing the effectiveness of various methods of eliminating space debris.

\subsection{Individual protection methods}

Includes shielding of the most important parts of the spacecraft, as well as evasive maneuvers (which is associated with the cost of the reactive mass). With the increasing amount of space debris, of these methods of protecting a spacecraft will become increasingly important ${ }^{1}$.

\subsection{Formulation of the problem}

As of July 2013, $\approx 29$ thousand fragments more than $10 \mathrm{~cm}, \approx 670$ thousand fragments from 1 to $10 \mathrm{~cm}, \approx 170$ million fragments from $1 \mathrm{~mm}$ to $1 \mathrm{~cm}$ rotated around the Earth [23]. The size of a single particle of space debris almost does not affect the probability of its entry into an operating

\footnotetext{
${ }^{1}$ It may be more cost-effective to insure spacecraft against colliding with space debris. Insurance can be attributed to the methods of protection (non-physical). Some of the funds accumulated by insurance companies will be used to implement large-scale space debris elimination projects (in order to reduce insurance payments). Another method of financing the fight against space debris could be a tax on activities in outer space.
} 
spacecraft. There are few large and medium-sized debris in orbit, they are clearly visible, their orbits are easy to predict. Therefore, they pose a threat to astronautics mainly as sources of small debris (because of collisions and fragmentation into small parts). At the same time, it is almost impossible to keep track of small space debris (which makes him very dangerous).

This article discusses the universal method of dealing with space debris, which consists in the correction of the orbits of cataloged debris and the destruction of unobservable particles of small debris. These tasks are supposed to be solved with the help of the orbital grouping of controlled micro-robots. Within the framework of this concept, some additional capabilities of space microrobots (orbit correction, braking or acceleration of spacecraft ${ }^{2}$ ) will be considered.

\section{Materials and Methods}

To combat space debris, it is supposed to use a large number of controlled micro-robots, which are displayed in a retrograde orbit (against the direction of the Earth's rotation) ${ }^{3}$. Because of a collision with micro-robots, the particles of space debris will leave the near-earth orbit (and the safety of the operating spacecraft's will be provide with a number of protective measures). Each micro-robot will have micro-engines, a power supply system (photocell, battery or other) and microcomputer, self-destruct system (detonator, explosive charge), devices for receiving and transmitting radio signals (radar, active transponder, and communications system), etc.

Micro-robots are made from highly volatile and combustible materials (and the explosive charge contains an excess of oxidizer) ${ }^{4}$. To activate the self-destruction system, a radio transmitter of the spacecraft is used, which emits a weak radio signal (encrypted). This signal is fixed by a micro-robot only at a very close distance (and then the micro-robot will turn into a harmless cloud of gas). Another way is to equip the micro-robot with a micro emitter and an echo sensor. If such a «radar» is sensitive enough, the micro-robot will undermine just before the collision.

An important precaution is to reduce the specific gravity of the micro-robot (for example, the hull can be made of penografit or others). The impact pressure is proportional to the density of the impactor [24]. Reducing the specific mass of the micro-robot minimizes possible harm in case of hit with the spacecraft.

As a micro-jet engine, a micro-capacity with compressed gas and special adjustable orifices is used. With the release of gas through these holes will create a jet thrust. Instead of gas, onecomponent rocket fuel (for example, hydrogen peroxide) can be stored in this micro-capacity. In this case, a catalyst is installed in the reactive micro-nozzles, and when hydrogen peroxide is released, it decomposes with the release of heat (creating reactive thrust). Another way is to use electric heating elements located on the surface of the micro-robot. These elements are coated with a solid substance that is able to sublimate when heated. As a result, by passing an electric current, jet thrust will be created. For testing, can be install different types of micro-jet engines on different series of micro-robots.

\footnotetext{
${ }^{2}$ With the help of a special engine, the principle of which is based on the capture of micro-robots.

${ }^{3}$ The main method of launching micro-robots into outer space is the use of rockets. However, the small size of micro-robots allows us to consider other methods (for example, a cannon scheme). In addition, at a high altitude (mountain, airship, plane, etc.) can position the accelerator, and accelerate and launch micro-robots using electromagnetic fields.

${ }^{4}$ Part of the oxidizer may react with the matter of space debris (which will partially evaporate). In the course of further research, it is necessary to model an ultrafast collision of an explosive micro-particle with a solid surface.
} 


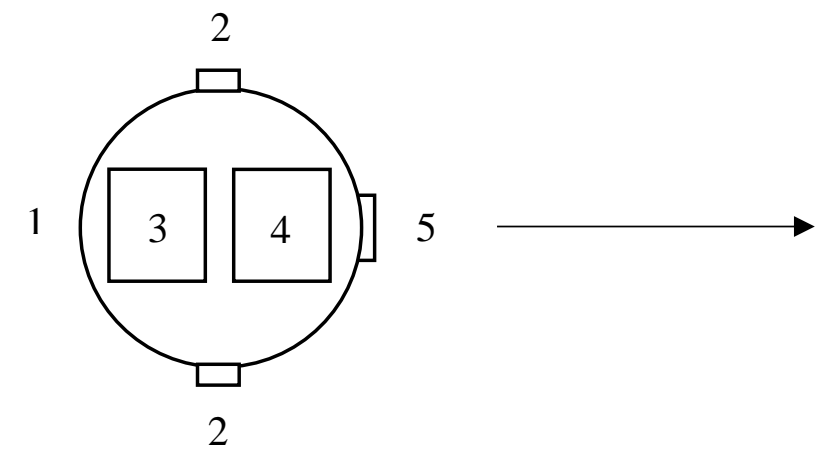

Figure 1. Schematic diagram of the micro-robot: 1 - hull; 2 - micro-jet engines; 3 - power supply system and microcomputer; 4 self-destruct system; 5 - devices for receiving and transmitting radio signals

To influence the cataloged objects of space debris, the micro-robots control system will be use. This system directs micro-robot on a space object (for to change its orbit, partial or complete destruction). Collisions of micro-robots with small space debris will occur randomly.

\section{1. Productivity calculation}

Let us determine the effectiveness of the proposed system (without taking into account the possibility of controlling micro-robots). The control will significantly increase the effectiveness of the system (therefore, this calculation of efficiency is an estimate of the lower limit). Suppose that a particle of debris moves in a circular orbit of radius $R$ (deceleration of a particle of space debris occurs very slowly, and its orbit can be considered as circular at any time). The oncoming speed of a particle of space debris and micro-robot is equal to $v$. The mass of the micro-robot is equal to $M_{r}$, the mass of the particle of space debris is equal to $M_{d}$. According to the law of conservation of momentum (with an inelastic central collision), it is obtained:

$$
M_{d} d v=-v M_{r}
$$

For an infinitely small period of time $d t$, the counter speed changes by the magnitude $d v$. Using equation (1) can be write the loss of total energy $d E$ particles of space debris:

$$
d E=M_{d} \frac{d v}{d t} v d t=-v^{2} M_{r}
$$

When the orbit height decreases by $d R$, the change in kinetic energy $d E_{k}$ is equal to:

$$
d E_{k}=-\frac{1}{2} M_{d} G M \frac{d R}{R^{2}}
$$

The change in potential energy $d E_{p}$ is equal to:

$$
d E_{p}=M_{d} G M \frac{d R}{R^{2}}
$$

According to the law of conservation of energy, using equations $(2 ; 3 ; 4)$ can be write: 


$$
d E=d E_{k}+d E_{p}=\frac{1}{2} M_{d} G M \frac{d R}{R^{2}}=-v^{2} M_{r}
$$

Taking into account that $v=2 v_{d}=2 v_{1}\left(v_{1}-\right.$ the first cosmic velocity), and using the equation of the first cosmic velocity can be rewrite equation (5) in the form:

$$
\frac{d R}{R}=-8 \frac{M_{r}}{M_{d}}
$$

Suppose that $R_{i}-$ is the initial radius of the orbit, $R_{f}-$ is the final radius of the orbit, $M_{r} \rightarrow 0$. Summing up the infinitesimal increments in equation (6) it will be obtained:

$$
\ln \frac{R_{i}}{R_{f}}=8 \frac{\sum M_{r}}{M_{d}}
$$

Suppose the average counter speed is $\bar{v}$, the debris particle cross-sectional area is $\Delta S$. Over a period of time $t$, micro-robots with a total mass of $\sum M_{r}$ will collide with a particle of space debris:

$$
\sum M_{r}=\Delta S N \bar{v} t M_{r}
$$

Suppose that micro-robots move within an orbital corridor of volume $V$, and have a total mass $M$ :

$$
M=N V M_{r}
$$

Assume that the concentration of micro-robots $N$ is constant $(N=$ Const). To characterize a particle of space debris, can be use the parameter $x$ (the ratio of the cross-sectional area ${ }^{5} \Delta S$ of particle to its own mass $M_{d}$ ):

$$
x=\frac{\Delta S}{M_{d}}
$$

Using equations $(9 ; 10)$, it is obtained:

$$
\frac{\sum M_{r}}{M_{d}}=x \bar{v} t \frac{M}{V}
$$

Using equations $(7 ; 11)$, it is obtained:

$$
\frac{R_{i}}{R_{f}}=e^{8 x \bar{v} t \frac{M}{V}}
$$

\section{2. Using the orbital grouping of micro-robots as an astronomical instrument and navigation system}

Suppose the concentration of micro-robots is $N$, the radar range of a micro-robot is $L$. When a particle of space debris and a micro-robot approaching the distance $L$ «micro-radar» can react, and transmit information to the control center on Earth. Can be consider as conditional "collision" the convergence of a micro-robot and particles of space debris at a distance of $L$. Using equation (8) can write the average amount of "collisions" $n \overline{\text { : }}$

$$
\bar{n} \approx \frac{\sum M_{r}}{M_{r}}=\Delta S N \bar{v} t
$$

\footnotetext{
${ }^{5}$ The area of the projection of a particle of space debris on a plane perpendicular to the direction of its movement.
} 
Considering that in this case conditional cross-sectional area of space debris particles $\Delta S=\pi L^{2}$, using equation (13), it is obtained the frequency $\omega$ of "collisions" (or the frequency of measurements of the coordinates of particle of space debris):

$$
\omega=\frac{\bar{n}}{t}=\pi L^{2} N \bar{v}=\pi L^{2} \frac{M}{V M_{r}} \bar{v}
$$

According to the dynamics of changes in the reflected signal, it is possible to establish not only the fact of approach, but also speed, minimum distance, etc. After receiving the information, the control center connect with the micro-robot for clarify its orbit and coordinates (at the time of signal transmission). Since the orbits of the cataloged particles are known, it is possible to determine which of them could be in a given region of near-Earth space. Then the coordinates of these particles are calculated (at the time of receiving the signal). Analysis of these data will allow us to identify a particle of space debris, and to clarify its orbital data.

In the event of significant discrepancies between the calculations and observations, it can be assumed that a new object has been discovered. In this case, the object is considered unidentified, and the information obtained is entered into a separate database. As data is accumulated in this database, an analysis is made that will allow determining the orbital data of new objects. After that, information about new objects can be entered into a general directory. Thus, systematic observation (with the help of the orbital grouping of micro-robots) will make it possible to control the orbits of space debris particles, and to search for new objects (of artificial and natural origin). This work will be most effective in cooperation with existing systems for the search and control of particles of space debris (telescopes, radar, etc.).

\section{3. Method of disposal of old micro-robots (or using micro-robots for braking or acceleration a spacecraft)}

Micro-robots can be used to change the orbit of a spacecraft (and performing the function of orientation engines) ${ }^{6}$. For this purpose, a control system is used, with the help of which the micro-robot is headed for the spacecraft ${ }^{7}$. Before the collision, the micro-robot turns into a harmless cloud of gas, and the spacecraft will receive a soft push, which corrects its orbit.

Over time, there may be a problem of disposal of old micro-robots in Earth orbit. One way to solve this problem is to create an engine that will use micro-robots to accelerate a spacecraft. Such an engine consists of a chamber with an inlet (on which a controllable flap is installed) ${ }^{8}$ and an outlet with a jet nozzle. Suppose a spacecraft and micro-robot are moving along one straight line (towards each other). Such an opportunity appears due to the use of the micro-robot control system. Thus, a large number of micro-robots are located along calculated the flight path of the spacecraft. The micro-robot gets into the inlet and explodes inside the working chamber. At the same time, a portion of the gas is fed into the working chamber. A uniformly heated gas mixture is formed, which flows to the right through the outlet (and the inlet is closed). Can show that the thrust impulse can exceed the deceleration impulse, and the spacecraft will receive an increase in the impulse to the left ${ }^{9}$.

\footnotetext{
${ }^{6}$ After completion of operation by this method, can slow down the spacecraft in order to burn it in the atmosphere of the Earth.

${ }^{7}$ In fact, the orbital grouping of micro-robots can be used as an anti-satellite weapon (when the self-destruction system is disabled).

${ }^{8}$ The function of flap can perform solenoid. For this, it is necessary to add easily ionizable substances (for example, alkali metals) to the gas.

${ }^{9}$ The existing literature describes promising engines that use the same principle of acceleration of spacecraft due to kinetic energy [25; 26].
} 


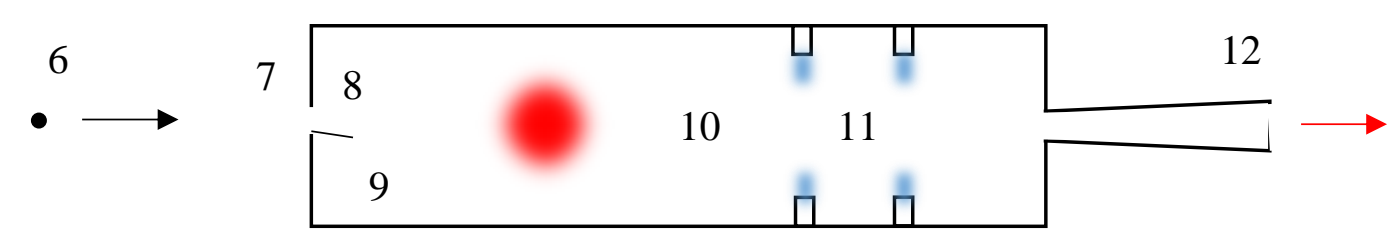

Figure 2. Schematic diagram of a jet engine using micro-robots: 6-micro-robot; 7-engine's working chamber; 8-inlet; 9flap at the inlet (e.g. solenoid); 10 - fireball (a cloud of gas formed during the explosion of a micro-robot); 11 - nozzles for supplying gas to the working chamber; 12 -jet nozzle outlet

Consider this event in the coordinate system of the spacecraft. The mass of the spacecraft $m$, the mass of the gas portion (supplied to the working chamber) is equal to $d M$, the exhaust speed of the gas mixture (from the working chamber) is equal to $u$. The expiration of the gas mixture (jet stream) occurs exactly along the tangent to the trajectory of the spacecraft. Engine efficiency is $\eta$. Using the law of conservation of energy (taking into account the specific energy $Q_{s p}$ the explosion of the micro-robot), it is obtained:

$$
\eta M_{r}\left(v^{2}+2 Q_{s p}\right)=\left(M_{r}+d M\right) u^{2}
$$

Next, can be move into the coordinate system rigidly connected with the micro-robot. The observed speed of jet stream in the coordinate system of the micro-robot is $(v-u)$. According to the law of conservation of momentum, it is obtained:

$$
m v=(m-d M)(v+d v)+\left(M_{r}+d M\right)(v-u)
$$

Suppose, $M_{r}=n d M$ ( $n$ - coefficient of proportionality), $g_{0}$ - the standard acceleration due to gravity on the surface of the Earth. Solving the system of equations $(13 ; 14)$ obtain a specific impulse $I_{s p}(\mathrm{~s})$ :

$$
I_{s p}=\frac{m d v}{d M} \frac{1}{g_{0}}=\frac{v}{g_{0}}\left(\sqrt{\eta n\left(1+\frac{2 Q_{s p}}{v^{2}}\right)(n+1)}-n\right)
$$

Using equation (17) can be write the condition of engine workability $\left(I_{s p}>0\right)$ in the form:

$$
n<\frac{\eta\left(1+\frac{2 Q_{s p}}{v^{2}}\right)}{1-\eta\left(1+\frac{2 Q_{s p}}{v^{2}}\right)}
$$

The maximum specific impulse $\left(I_{s p}\right)_{\max }$ achieved in the case of $n=n_{\text {opt }}$. Using the equation (17) can write: 


$$
\left(\sqrt{\eta n\left(1+\frac{2 Q_{s p}}{v^{2}}\right)(n+1)}-n\right)^{\prime}=0
$$

Solving an equation (19) for a variable $n$ it is obtained:

$$
n_{\text {opt }}=\frac{1}{2}\left(\frac{1}{\sqrt{1-\eta\left(1+\frac{2 Q_{s p}}{v^{2}}\right)}}-1\right) \quad \text { on condition } \eta\left(1+\frac{2 Q_{s p}}{v^{2}}\right)<1
$$

Consider the case when $2 Q_{s p} / v^{2} \rightarrow 0$. Suppose $v_{i}$-initial oncoming speed, $v_{f}$ - final oncoming speed, $m_{i}$ - initial mass the spacecraft, $m_{f}$ - final mass of the spacecraft. Considering that $d M=-$ $d m$, and summing up the infinitesimal increments in equation (17), on condition $\eta=$ Const, get:

$$
\frac{v_{i}}{v_{f}}=\left(\frac{m_{f}}{m_{i}}\right)^{\sqrt{\eta n(n+1)}-n}
$$

As the height increases, the oncoming speed $v$ decreases. Therefore, it is advisable to accelerate the spacecraft in several stages, including the engine at the time of passage of the perigee. The orbital period $T_{o}$ (for an elliptical orbit) depends on the semi-major axis $a$, and the gravitational parameter $\mu$ :

$$
T_{o}=2 \pi \sqrt{\frac{a^{3}}{\mu}}
$$

The speed of the spacecraft $v_{s}$ at the pericenter depends on the distance $R_{p}$ and is equal to:

$$
v_{s}=\sqrt{\mu\left(\frac{2}{R_{p}}-\frac{1}{a}\right)}
$$

The second cosmic velocity is $v_{2}$. Using equations $(22 ; 23)$ can be write:

$$
T_{o}=2 \pi \mu \sqrt{\frac{1}{\left(v_{2}^{2}-v_{s}^{2}\right)^{3}}}
$$

To determine the coordinates can be install navigation modules on micro-robots or use a secondary surveillance radar. The secondary radar provides for installation of transponders on micro-robots. RFID-tags (an element of the radio-frequency identification system) can be used as transponders. The RFID-tag will transmit the unique identification number of each micro-robot. Existing RFID-tags may have a characteristic size of $\approx 0.05 \mathrm{~mm}[27]$; cost $\approx 5$ cents per piece [28]. However, for space more sophisticated and expensive FRID-tags will be need. Using a secondary surveillance radar using RFID-tags will make it possible to determine the coordinates and speed of micro-robots (and calculate the trajectory of motion). To increase the efficiency, it is advisable to launch small probe-spotter equipped with secondary radar ahead of the spacecraft (Fig. 3). Self-organization of micro-robots through the exchange of information (the concept of smart dust) is also a promising direction. At the implementation of this concept, micro-robots will be able to line up independently along the calculated flight path of the spacecraft. 


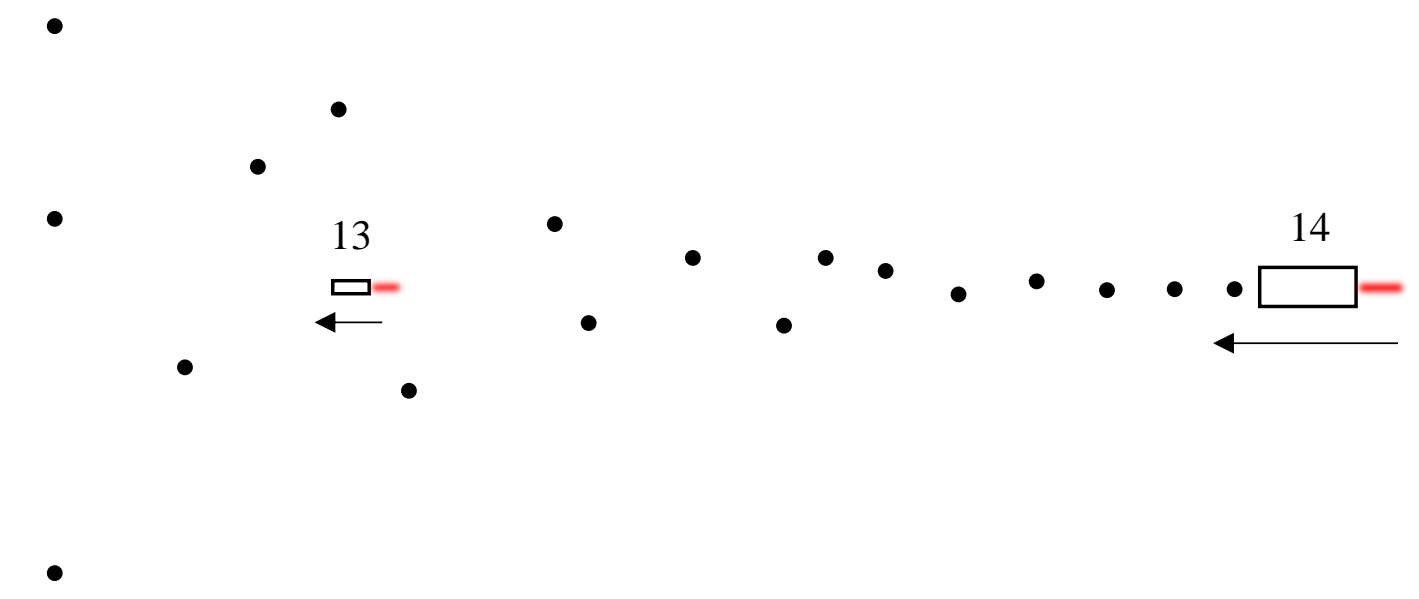

Figure 3. Schematic diagram of the acceleration of the spacecraft, using the probe-spotter: 13 - probe-spotter (with a radar system); 14 - spacecraft (also equipped with a radar system)

\section{Results}

\subsection{Specifications orbital groupings of micro-robots and calculating her effectiveness (for removing space debris and for detecting particles of space debris)}

Suppose a micro-robot is a sphere with a radius of $2 \mathrm{~mm}$ and a mass of $M_{r}=10 \mathrm{mg}$. Consider the task of cleaning the orbital corridor with the following parameters: height $\approx 600-1000 \mathrm{~km}$, width $\approx 1$ thousand $\mathrm{km}$, circumference $\approx 45$ thousand $\mathrm{km}$. Its volume is $V \approx 1.8 \cdot 10^{19} \mathrm{~m}^{3}$.

The value of the parameter $x$ is significantly different for different particles of space debris. For example, for a cataloged space object № $95334 x \approx 2.2 \mathrm{~m}^{2} / \mathrm{kg}$, for a space object № $90073 x \approx$ $0.18 \mathrm{~m}^{2} / \mathrm{kg}$ [29]. Therefore, it is necessary to use the averaged value of the parameter $x$. Consider a fraction of space debris with a size of $1 \mathrm{~mm}-1 \mathrm{~cm}$, as the most numerous according to the ESA [22]. Assume an average characteristic particle size of $\approx 5 \mathrm{~mm}$. If simplified to consider a small particle of space debris as an aluminum sphere, then its midsection section $x \approx 0.11 \mathrm{~m}^{2} / \mathrm{kg}$.

Suppose that $t=10$ years, $R_{i}=7400 \mathrm{~km}, R_{f}=7000 \mathrm{~km}, \bar{v}=14.9 \mathrm{~km} / \mathrm{s}, x \approx 0.11 \mathrm{~m}^{2} / \mathrm{kg}$. Using equation (12) it is obtain the required mass of micro-robots $M=242$ tons (for the almost complete elimination of small space debris from the orbital corridor). The cost of delivering $1 \mathrm{~kg}$ of cargo to a low earth orbit using the launch vehicle «Proton-M» is $\$ 2,826$ [30]. With this in mind, the launch cost of 242 tons of micro-robots can be approximately estimated at $\$ 684$ million. However, this amount may be more, since the launch of micro-robots is carried out against the direction of rotation of the Earth (which will reduce the payload of the launch vehicle).

Based on the data obtained, can be determine the frequency of detection of particles of space debris (or meteoroids). Supposing $M=242$ tons, $M_{r}=10 \mathrm{mg}, V=1.8 \cdot 10^{19} \mathrm{~m}^{3}, \bar{v}=14.9 \mathrm{~km} / \mathrm{s}$. Using equation (14) will get $\omega=6.3 \cdot 10^{-5} \mathrm{~Hz}$ (or about $5-6$ measurements during the twentyfour hours for each particles of space debris). It is obvious that such a measurement frequency makes it possible with high accuracy and reliability to control space debris in Earth orbit. In addition, such a system allows not only determining the coordinates of particles of space debris, 
but also the coordinates of micro-robots. This simplifies the task of targeting micro-robots to medium and large particles of space debris.

\section{2. Calculation of the efficiency of the engine using micro-robots}

A significant part of micro-robots will not be able to collide with space debris. Over time, they will end up at in low orbits (as a result, of braking by the upper layers of the atmosphere). Consider an example the use of a new engine as a convenient disposal method of old microrobots. Suppose the orbit pericenter is located at a distance of $200 \mathrm{~km}$ to the Earth's surface, the first cosmic velocity $v_{1}=7.8 \mathrm{~km} / \mathrm{s}$, the second cosmic velocity $v_{2}=11 \mathrm{~km} / \mathrm{s}$, the Earth's gravity parameter $\mu=4 \cdot 10^{14} \mathrm{~m}^{3} / \mathrm{s}^{2}$. The acceleration impulse is carried out in the pericenter of the orbit of the spacecraft. The total time spent consists of the total duration of the spacecraft moving in elliptical orbits, according to equation (24).

Table 1

Dynamics of the acceleration of the spacecraft

\begin{tabular}{cccccccc}
\hline Parameter & \multicolumn{4}{c}{ The number of the acceleration pulse } & \multicolumn{2}{c}{ Overall } \\
& 1 & 2 & 3 & 4 & 5 & 6 & result \\
\hline Speed increase, $\mathrm{m} / \mathrm{s}$ & 600 & 600 & 600 & 600 & 600 & 600 & 3600 \\
Rotation period $^{10}, \mathrm{~s}$ & - & 6880 & 9694 & 15685 & 33977 & - & 66236 \\
\hline
\end{tabular}

From Table 1 it can be seen that the overall speed increase of the spacecraft will be $3.6 \mathrm{~km} / \mathrm{s}$. The final speed of the spacecraft $v_{s}=11.4 \mathrm{~km} / \mathrm{s}$ allows flight to Mars [31]. The total time spent on the rotation of the spacecraft in elliptical orbits is $\sum T o=18.4$ hours. Cyclic engine operation will reduce the heat load (due to the periodic cooling of the working chamber).

It is necessary to determine the specifications of the engine. The initial oncoming speed (microrobots and spacecraft) is equal to $v_{i}=15.6 \mathrm{~km} / \mathrm{s}(7.8 \mathrm{~km} / \mathrm{s}+7.8 \mathrm{~km} / \mathrm{s})$. The final oncoming speed is $v_{f}=19.2 \mathrm{~km} / \mathrm{s}(7.8 \mathrm{~km} / \mathrm{s}+11.4 \mathrm{~km} / \mathrm{s})$. Suppose that $Q_{s p}=0, \eta=0.6$. Using equation (20) it is obtained $n_{\text {opt }}=0.29$. Using equation (21) it is obtained $m_{i} / m_{f}=3.1$. Substituting this value into the Tsiolkovsky rocket equation, it is obtained the equivalent value of the specific impulse $I_{s p}=$ $325 \mathrm{~s}$ (by the same procedure, provided $\eta=0.8$, it is obtained $I_{s p}=488 \mathrm{~s}$ ). It should be noted that with the use of such a way to accelerate the spacecraft near the giant planets, the specific impulse would increase several times (due to the greater counter-velocity of the spacecraft and microrobots). It is possible that during the colonization of giant planets, there will also be a problem of cleaning the near-planetary space (for example, from natural space debris). In this case, the use of micro-robots can be a convenient method for solving two problems simultaneously: eliminating natural space debris and accelerating spacecraft.

Such a specific impulse value is comparable to the specific impulse of the best liquid-fuelled rocket engines. The principle of operation of the new engine makes it possible to use very dense substances (for example, xenon) as the reactive mass. The density of liquid xenon is much higher than the density of rocket propellant, which saves weight by reducing the size of tanks. In addition, increased flight safety, due to the absence of a potential threat of rocket propellant.

Suppose the initial mass of a spacecraft is $m_{i}=1000 \mathrm{~kg}$, the initial speed is $v_{i}=7.8 \mathrm{~km} / \mathrm{s}$ (in the coordinate system of the Earth), the kinetic energy is $\approx 30.4$ GJ. From the above example, it is seen that the final mass of the spacecraft is $m_{f}=323 \mathrm{~kg}$ (since $m_{i} / m_{f}=3.1$ ), the final speed is $v_{f}=$ $11.4 \mathrm{~km} / \mathrm{s}$ (in the coordinate system of the Earth), the kinetic energy is $\approx 21 \mathrm{GJ}$. Thus, in the

\footnotetext{
${ }^{10}$ The duration of acceleration is the time interval between the first and last acceleration pulses (therefore, the duration of the first and last periods of rotation are not taken into account).
} 
process of acceleration, the kinetic energy of the spacecraft decreased to $69 \%$ of the initial level. This fact indicates that the new engine is not Perpetuum Mobile (as it may seem at first glance).

Let us assume that the velocity of propagation of a blast wave in an explosive is $\approx 4 \mathrm{~km} / \mathrm{s}$. Since the characteristic size of a micro-robot is $4 \mathrm{~mm}$, the self-destruction time is approximately $\approx 10^{-6}$ s. During this time, the micro-robot will fly $\approx 2 \mathrm{~cm}$ (at a speed of $19.2 \mathrm{~km} / \mathrm{s}$ ). Thus, the use of micro-robots does not impose significant restrictions on the minimum size of the working chamber of the engine. In case of successful creation of a new engine, one of its main indicators will be its clock frequency (the number of micro-robots that explode inside the working chamber per unit of time).

Supposing, a probe-spotter moves in front of a spacecraft at a distance of 5 thousand $\mathrm{km}$. To overcome this distance with a medium counter speed of $17.6 \mathrm{~km} / \mathrm{s}$, the micro-robot needs time $\approx$ $284 \mathrm{~s}$. Supposing, a micro-engines of a micro-robot provide acceleration of $\approx 1 \mathrm{~m} / \mathrm{s}^{2}$. Over the specified time, the trajectory of the micro-robot can deviate in the right direction to a distance of up to $\approx 40.3 \mathrm{~km}$, and the cross-sectional area (of space corridor from which micro-robots are collected) will be $\Delta S \approx 5.1$ thousand $\mathrm{km}^{2}$. Assume $M=242 \mathrm{t}, V=1.8 \cdot 10^{19} \mathrm{~m}^{3}, \bar{v}=17.6 \mathrm{~km} / \mathrm{s}$. Using equations ( $8 ; 9$ ), can be obtain the mass flow of micro-robots $\approx 1.2 \mathrm{~kg} / \mathrm{s}$, the engine clock frequency $\approx 120 \mathrm{kHz}$. On condition $I_{s p}=488 \mathrm{c}$, the average engine thrust force will be $\approx 5745 \mathrm{~N}$.

\section{Conclusions}

The article describes a new method of dealing with space debris, which consists in using controlled micro-robots moving in a retrograde near-earth orbit towards space debris particles. It is shown that the use of controlled micro-robots is an effective and safe method of dealing with space debris. The use of space micro-robots allows you to eliminate small space debris from near-Earth orbit and control the movement of medium and large particles of space debris. This prevents the development of events according to the Kessler scenario (uncontrolled division of space debris). In addition, the development and use of controlled space micro-robots is a promising direction for the development of space technologies, which is at the junction of several important problems of cosmonautics. For example, controlled micro robots can be used to accelerate, decelerate, and correct the orbit of a spacecraft. Orbital grouping of micro-robots can be used to search and refine the coordinates of space debris particles (or space particles of natural origin). Based on controlled micro-robots, it is also possible to develop and create a new engine to accelerate the spacecraft. Such an engine is highly efficient and can be used for flights to other planets of the solar system (for example, to Mars).

\section{On behalf of all authors, the corresponding author states that there is no conflict of interest.}

\section{References}

[1] Donald J. Kessler, Burton G. Cour-Palais, Collision frequency of artificial satellites: The creation of a debris belt, JGR Space Physics, Volume83, Issue A6, June 1978, Pp 2637-2646, https://doi.org/10.1029/JA083iA06p02637

[2] Kerry T. Nock, Kristin L. Gates, Kim M. Aaron, and Angus D. McRonald, Gossamer Orbit Lowering Device (GOLD) for Safe and Efficient De-orbit, AIAA/AAS Astrodynamics Specialist Conference 2 - 5 August 2010, Toronto, Ontario Canada, DOI: 10.2514/6.2010-7824 
[3] Trofimov S. P. Disposal of small spacecraft from low near-earth orbits: dissertation by Cand. Phys.-Mat. Sciences: 01.02.01. M., 2015. 125 p. URL:

http://library.keldysh.ru/diss.asp?id=2015-trofimov

[4] Patrick W. Kelly, Riccardo Bevilacqua, Leonel Mazal, and Richard S. Erwin. "TugSat: Removing Space Debris from Geostationary Orbits Using Solar Sails", Journal of Spacecraft and Rockets, Vol. 55, No. 2 (2018), pp. 437-450. https://doi.org/10.2514/1.A33872

[5] P. Pergola, A. Ruggiero, and M. Andrenucci, Low-thrust Missions for Expanding Foam Space Debris Removal, The 32-nd International Electric Propulsion Conference, Wiesbaden, Germany, September 11 - 15, 2011, http://erps.spacegrant.org/uploads/images/images/iepc articledownload 19882007/2011index/IEPC-2011-126.pdf

[6] Research and Development Directorate pamphlet, Japan Aerospace Exploration Agency, 2016, http://www.kenkai.jaxa.jp/eng/publication/pamphlet/pdf/RD2016_eng.pdf

[7] Kanjuro Makihara and Shu Kondo. "Structural Evaluation for Electrodynamic Tape Tethers Against Hypervelocity Space Debris Impacts", Journal of Spacecraft and Rockets, Vol. 55, No. 2 (2018), pp. 462-472. https://doi.org/10.2514/1.A34023

[8] Schaub H., Sternovsky Z. Active space debris charging for contactless electrostatic disposal maneuvers // Advances in Space Research, 2014, vol. 53, no.1, pp. 110 - 118. DOI:

10.1016/j.asr.2013.10.003

[9] Benvenuto R., Salvi S., Lavagna M. Dynamics analysis and GNC design of flexible systems for space debris active removal // Acta Astronautica, 2015, vol. 110, pp. 247 - 265. DOI:

10.1016/j.actaastro.2015.01.014

[10] Sharf I., Thomsen B., Botta E.M., Misra A.K. Experiments and simulation of a net closing mechanism for tether-net capture of space debris // Acta Astronautica, 2017, vol. 139, pp. 332 343. https://doi.org/10.1016/j.actaastro.2017.07.026

[11] Siegfried W. Janson, Brane Craft (A 2016-2017 NIAC Phase I Project) Final Report Submitted to NASA on February 1, 2017,

https://ntrs.nasa.gov/archive/nasa/casi.ntrs.nasa.gov/20170002796.pdf

[12] Forshaw J.L., Aglietti G.S., Salmon T., Retat I., Roe M., Burgess C., Chaumette F. Final payload test results for the Remove Debris active debris removal mission // Acta Astronautica, 2017, vol. 138, pp. 326 - 342. https://doi.org/10.1016/j.actaastro.2017.06.003

[13] Matt Williams, China has a plan to clean up space junk with lasers, Universe Today, January 17, 2018 https://phys.org/news/2018-01-china-space-junk-lasers.html

[14] Phipps C.R., Bonnal C. A spaceborne, pulsed UV laser system for re-entering or nudging LEO debris, and re-orbiting GEO debris // Acta Astronautica, 2016, vol. 118, pp. 224 - 236, DOI: $10.1016 /$ j.actaastro.2015.10.005

[15] Phipps C.R. A laser-optical system to re-enter or lower low Earth orbit space debris // Acta Astronautica, 2014, vol. 93, pp. 418 - 429, DOI: 10.1016/j.actaastro.2013.07.031

[16] S. V. Khoroshylov, A.P. Alpatov, NON-CONTACT REMOVAL OF SPACE WASH BY ION BEAM. Dynamics and control, Book: June 2018, ISBN 978-613-9-81969-0, Publisher: LAP Lambert Academic Publishing RU, https://www.researchgate.net/publication/325995367_BESKONTAKTNOE_UDALENIE_KOS MICESKOGO MUSORA IONNYM LUCOM Dinamika i upravlenie 
[17] Kazunori Takahashi, Christine Charles, Rod W. Boswell \& Akira Ando, Demonstrating a new technology for space debris removal using a bi-directional plasma thruster, Scientific Reports, Volume 8, Article number: 14417 (2018), DOI: 10.1038/s41598-018-32697-4

[18] By Gurudas GANGULI, Christopher CRABTREE, Leonid RUDAKOV, and Scott CHAPPIE, A Concept For Elimination Of Small Orbital Debris, 2011, https://arxiv.org/ftp/arxiv/papers/1104/1104.1401.pdf

[19] Lei Lan, Jingyang Li, Hexi Baoyin, Debris Engine: A Potential Thruster for Space Debris Removal, 2015, https://arxiv.org/abs/1511.07246

[20] Dmitry Novoseltsev, On the possibility of rational utilization of fragments of near-Earth space debris and meteoroid matter using kinetic rocket engines, NAKED SCIENCE (20 September 2018), https://naked-science.ru/article/column/o-vozmozhnosti-racionalnoy

[21] Emiko Jozuka, This Satellite Uses Special Glue to Stick to Space Debris Like Flypaper, Motherboard, May 2, 2016, https://motherboard.vice.com/en us/article/aeke5a/this-satelliteuses-special-glue-to-stick-to-space-debris-like-flypaper

[22] Minghe Shan, Jian Guo, Eberhard Gill, Review and comparison of active space debris capturing and removal methods // Progress in Aerospace Sciences, 2016, vol. 80, pp. 18 - 32. https://doi.org/10.1016/j.paerosci.2015.11.001

[23] How many space debris objects are currently in orbit?" ESA, July 2013, http://www.esa.int/Our_Activities/Space_Engineering_Technology/Clean_Space/How_many_sp ace_debris_objects_are_currently_in_orbit

[24] M. M. Rusakov. Equivalence of a high-speed impact by a bunch of tungsten particles on obstacles and collisions with real cosmic particles URL:

http://www.vniitf.ru/rig/konfer/8zst/s1/1-30.pdf

[25] V. V. Podvysotsky, Theoretical Study of the Possibility of Creating Kinetic Jet Engine, SCIENCE PROSPECTS, 4 (2013), pp. 56-66. URL:

http://moofrnk.com/assets/files/journals/science-prospects/43/science-prospects-4(43)-2013.pdf

[26] Valentin Vladimirovich Podvysotsky, Superconductors Applied in Electrodynamic Engine, International Review of Aerospace Engineering 11(3):120 June 2018 DOI:

10.15866/irease.v11i3.14601

[27] TFOT, Hitachi Develops World's Smallest RFID Chip, 2007 URL:

https://thefutureofthings.com/3221-hitachi-develops-worlds-smallest-rfid-chip/

[28] Mark Roberti. A 5-Cent Breakthrough. RFID Journal. May 01, 2006 URL: https://www.rfidjournal.com/articles/view?2295

[29] Levkina Polina Anatolyevna, Physical and orbital characteristics of objects of space debris according to optical observations, Abstract of thesis for the degree of candidate of physical and mathematical sciences, Moscow, 2016 URL: http://www.gaoran.ru/russian/diss/LevkinaPA.pdf

[30] United States Government Accountability Office, Sale Price Drives Potential Effects on DOD and Commercial Launch Providers, August 2017, P. 30 URL:

https://www.gao.gov/assets/690/686613.pdf

[31] Levantovsky V. I. The mechanics of space flight in elementary presentation. 3-rd ed., Ext. and pererabat. - M.: Nauka, 1980. P. - 311. URL: http://books.sernam.ru/book_msp.php?id=97 